\title{
Positive blow-up solutions of nonlinear models from real world dynamics
}

\author{
Jürgen Gschwindl ${ }^{1}$, Irena Rachůnková2 ${ }^{*}$, Svatoslav Staněk² and Ewa B Weinmüller ${ }^{1}$
}

Dedicated to Professor Ivan Kiguradze for his merits in mathematical sciences

${ }^{*}$ Correspondence:

irena.rachunkova@upol.cz

${ }^{2}$ Department of Mathematics,

Faculty of Science, Palacký

University, 17. listopadu 12,

Olomouc, 77146, Czech Republic

Full list of author information is

available at the end of the article

\begin{abstract}
In this paper, we investigate the structure and properties of the set of positive blow-up solutions of the differential equation $\left(t^{k} v^{\prime}(t)\right)^{\prime}=t^{k} h(t, v(t)), t \in(0, T] \subset \mathbb{R}$, where $k \in(1, \infty)$. The differential equation is studied together with the boundary conditions $\lim _{t \rightarrow 0+} v(t)=\infty, v(T)=0$. We specify conditions for the data function $h$ which guarantee that the set of all positive solutions to the above boundary value problem is nonempty. Further properties of the solutions are discussed and results of numerical simulations are presented.
\end{abstract}

MSC: 34B18; 34B16; 34A12

Keywords: singular ordinary differential equation of the second order; time singularities; blow-up, positive solutions; existence of solutions; polynomial collocation

\section{Introduction}

In this paper, we investigate the structure and properties of the set of positive blow-up solutions of the differential equation

$$
\left(t^{k} v^{\prime}(t)\right)^{\prime}=t^{k} h(t, v(t)), \quad t \in(0, T] \subset \mathbb{R},
$$

where $k \in(1, \infty)$.

Models in the form of (1) arise in many applications. Among others, they occur in the study of phase transitions of Van der Waals fluids [1-3], in population genetics, where they characterize the spatial distribution of the genetic composition of a population $[4,5]$, in the homogeneous nucleation theory [6], in relativistic cosmology for particles which can be treated as domains in the universe [7], and in the nonlinear field theory, in particular, in context of bubbles generated by scalar fields of the Higgs type in Minkowski spaces [8].

Here, we assume that $h$ is positive and satisfies the Carathéodory conditions on $[0, T] \times$ $[0, \infty)$. We define a positive solution of (1) as a function $v$ which satisfies (1) for a.e. $t \in$ $[0, T]$, is positive on $(0, T)$, and has absolutely continuous first derivative on each compact subinterval in $(0, T]$.

According to Lemma 4, if $v$ is a positive solution of (1), then either

$$
\lim _{t \rightarrow 0+} v(t) \in[0, \infty), \quad \lim _{t \rightarrow 0+} v^{\prime}(t)=0,
$$

O2014 Gschwindl et al.; licensee Springer. This is an Open Access article distributed under the terms of the Creative Commons Attribution License (http://creativecommons.org/licenses/by/2.0), which permits unrestricted use, distribution, and reproduction in any medium, provided the original work is properly cited. 
or

$$
\lim _{t \rightarrow 0+} v(t)=\infty, \quad \lim _{t \rightarrow 0+} v^{\prime}(t)=-\infty
$$

In the literature, bounded solutions of (1) have been widely investigated; see e.g. [9-13]. Such solutions are characterized by the initial condition $\lim _{t \rightarrow 0+} v^{\prime}(t)=0$. In contrast to this, some real problems lead to the investigation of unbounded solutions which are characterized by the condition $\lim _{t \rightarrow t^{*}} v(t)=\infty$ for some $t^{*} \in[0, T]$ and which are called blowup solutions. We refer to [14-16]. Here, we are interested in blow-up solutions of (1), where $t^{*}=0$. In particular, (1) will be considered together with the boundary conditions

$$
\lim _{t \rightarrow 0+} v(t)=\infty, \quad v(T)=0
$$

In this case, we speak about a positive solution of problem (1), (2). Let us denote by $\mathcal{Z}$ the set of all positive solutions to (1), (2). Moreover, let $\mathcal{R}=\left\{v \in \mathcal{Z}: t^{k} v\right.$ is bounded on $\left.(0, T]\right\}$ and $\mathcal{R}_{c}=\left\{v \in \mathcal{R}: v^{\prime}(T)=-c\right\}$ for $c \geq 0$. Since $v^{\prime}(T) \leq 0$ for each $v \in \mathcal{Z}$, it is obvious that $\mathcal{R}=\bigcup_{c \geq 0} \mathcal{R}_{c}$.

Our main goal is to find conditions for the data function $h$ in (1), which guarantee that the set $\mathcal{R}_{c}$ is nonempty for each $c \geq 0$ and then to investigate the properties of this set. For example, we prove that the difference of any two functions in $\mathcal{R}_{c}, c \geq 0$, retains its sign on $[0, T)$, and that there exist minimal and maximal solutions $v_{c, \min }, v_{c, \max } \in \mathcal{R}_{c}$ for each $c \geq 0$, $c f$. Theorem 5. If the interior of the set $\left\{(t, x) \in \mathbb{R}^{2}: 0 \leq t \leq T, v_{c, \min }(t) \leq x \leq v_{c \text {,max }}(t)\right\}$ is nonempty, we show that this interior is fully covered by ordered graphs of other functions belonging to $\mathcal{R}_{c}$ for each $c \geq 0$; see Theorem 6 . Finally, in Theorem 7 , the existence of a positive constant $\alpha_{0}$ such that $\alpha_{0}=\lim _{t \rightarrow 0+} t^{k-1} v_{0, \min }(t)$ is shown and all functions $v$ from $\mathcal{R}$ are uniquely characterized by the condition

$$
\lim _{t \rightarrow 0+} t^{k-1} v(t)=\alpha, \quad \alpha \in\left[\alpha_{0}, \infty\right)
$$

If we denote such $v$ by $v_{\alpha}$ and define $w_{\alpha}(t)=t^{k-1} v(t)$ for $t \in(0, T]$, and $w_{\alpha}(0)=\alpha$, then we find that the graphs of these functions do not intersect, $c f$. Theorem 8 , and that for each $\alpha^{*}>\alpha_{0}$, the set $\left\{w_{\alpha}: \alpha_{0} \leq \alpha \leq \alpha^{*}\right\}$ is compact in $C[0, T]$; see Theorem 9 .

The study of a structure of positive solutions to other types of ordinary differential equations can be found for example in [17-19].

\section{Notation}

Let us denote by $C[0, T]$ the Banach space of functions continuous on $[0, T]$ equipped with the maximum norm

$$
\|x\|_{\infty}=\max \{|x(t)|: t \in[0, T]\} .
$$

Similarly, $C^{1}[0, T]$ means the Banach space of functions having a continuous first derivative on $[0, T]$ with the corresponding maximum norm $\|x\|_{\infty}+\left\|x^{\prime}\right\|_{\infty}$. By $L^{1}[0, T]$ we denote the set of functions which are Lebesgue integrable on $[0, T]$. Moreover, $A C^{1}[0, T]$ is the set of functions whose first derivative is absolutely continuous on $[0, T]$, while $A C_{\text {loc }}^{1}(0, T]$ is 
the set of functions having absolutely continuous first derivative on each compact subinterval of $(0, T]$. We say that $h(t, x)$ satisfies the Carathéodory conditions on $[0, T] \times[0, \infty)$, if the following three conditions hold:

(i) The function $h(\cdot, x):[0, T] \rightarrow \mathbb{R}$ is measurable for all $x \in[0, \infty)$.

(ii) The function $h(t, \cdot):[0, \infty) \rightarrow \mathbb{R}$ is continuous for a.e. $t \in[0, T]$.

(iii) For each compact set $\mathcal{U} \subset[0, \infty)$ there exists a function $m_{\mathcal{U}} \in L^{1}[0, T]$ such that

$$
|h(t, x)| \leq m_{\mathcal{U}}(t) \quad \text { for a.e. } t \in[0, T] \text { and all } x \in \mathcal{U} \text {. }
$$

For functions satisfying above conditions, we use the notation $h \in \operatorname{Car}([0, T] \times[0, \infty))$.

\section{Structure of the paper}

The paper is organized as follows. In Section 2 we discuss properties of the solutions of the auxiliary Dirichlet problem (3), (4). We recapitulate previous results from [20] and also present new results in Theorems 1,2, and 3. The main results of the paper can be found in Section 3, where we describe a relation between solutions of problem (3), (4) and blow-up solutions of problem (1), (2); see Theorem 4. Using this relation and the results of Section 2, we obtain various interesting properties of blow-up solutions; see Theorems 5 to 9 . Section 4 contains three examples illustrating the theoretical findings. Final remarks and open problems are formulated in Section 5.

\section{Auxiliary results}

In this section we consider the auxiliary singular differential equation,

$$
u^{\prime \prime}(t)+\frac{a}{t} u^{\prime}(t)-\frac{a}{t^{2}} u(t)=f(t, u(t))
$$

where $a<-1$. For $k:=-a$ and $v(t):=t^{-k} u(t)$, (3) becomes a special case of (1) and therefore, results obtained for (3) apply for (1). For the further analysis, we assume that $f$ satisfies the following conditions:

$\left(\mathrm{H}_{1}\right) f \in \operatorname{Car}([0, T] \times[0, \infty))$,

$\left(\mathrm{H}_{2}\right) \quad 0<f(t, x)$ for a.e. $t \in[0, T]$ and all $x \in[0, \infty)$,

$\left(\mathrm{H}_{3}\right) f(t, x)$ is increasing in $x$ for a.e. $t \in[0, T]$ and

$$
\lim _{x \rightarrow \infty} \frac{1}{x} \int_{0}^{T} f(t, x) \mathrm{d} t=0
$$

We now study (3) subject to the boundary conditions

$$
u(0)=0, \quad u(T)=0,
$$

and require that

$$
u^{\prime}(T)=-c, \quad c \geq 0,
$$

holds. We call a function $u:[0, T] \rightarrow \mathbb{R}$ a positive solution of the Dirichlet problem (3), (4) if $u \in A C^{1}[0, T], u>0$ on $(0, T), u$ satisfies the boundary conditions (4), and (3) holds for 
a.e. $t \in[0, T]$. Clearly, for each positive solution $u$ of (3), (4), there exists $c \geq 0$ such that (5) is satisfied.

We now denote by $\mathcal{S}$ the set of all positive solutions of problem (3), (4), and $\mathcal{S}_{c}=\{u \in$ $\left.\mathcal{S}: u^{\prime}(T)=-c\right\}$, where $c \geq 0$.

In the following lemma we cite those results from [20] which will be used in the analysis of problem (1), (2).

Lemma 1 Let $\left(\mathrm{H}_{1}\right)-\left(\mathrm{H}_{3}\right)$ hold. Then the following statements hold:

(a) For each $c \geq 0$ the set $\mathcal{S}_{c}$ is nonempty and there exist functions $u_{c, \min }, u_{c, \max } \in \mathcal{S}_{c}$ such that $u_{c, \min }(t) \leq u(t) \leq u_{c, \max }(t)$ for $t \in[0, T]$ and $u \in \mathcal{S}_{c}$.

(b) If $c_{1}>c_{2} \geq 0, u_{i} \in \mathcal{S}_{c_{i}}, i=1,2$, then $u_{1}(t)>u_{2}(t)$ for $t \in(0, T)$.

(c) If $c \geq 0, u_{i} \in \mathcal{S}_{c}, i=1,2$, and $u_{1}\left(t_{0}\right)>u_{2}\left(t_{0}\right)$ for some $t_{0} \in(0, T)$, then either $u_{1}(t)>u_{2}(t)$ for $t \in(0, T)$ or there exists $t_{*} \in\left(t_{0}, T\right)$ such that $u_{1}(t)>u_{2}(t)$ for $t \in\left(0, t_{*}\right)$ and $u_{1}(t)=u_{2}(t)$ for $t \in\left[t_{*}, T\right]$.

(d) For each $t_{0} \in(0, T)$ and each $A>u_{0, \max }\left(t_{0}\right)$ there exists $u \in \mathcal{S}$ satisfying $u\left(t_{0}\right)=A$.

(e) $\mathcal{S}_{c}$ is a one-point set for each $c \in[0, \infty) \backslash \Gamma$, where $\Gamma \subset[0, \infty)$ is at most countable.

(f) For each $0 \leq K \leq Q<\infty$, the set $\bigcup_{K \leq c \leq Q} \mathcal{S}_{c}$ is compact in $C^{1}[0, T]$.

(g) If $c \geq 0$, then $u \in \mathcal{S}_{c}$ if and only if it is a solution of the equation

$$
u(t)=t \frac{c T^{a+1}}{|a+1|}\left(T^{-a-1}-t^{-a-1}\right)+t \int_{t}^{T} s^{-a-2}\left(\int_{s}^{T} \xi^{a+1} f(\xi, u(\xi)) \mathrm{d} \xi\right) \mathrm{d} s
$$

in the set $C^{1}[0, T]$.

We now formulate new results which complete those from [20]. We first establish a relation between $\mathcal{S}_{0}$ and the set $\left\{(t, x) \in \mathbb{R}^{2}: 0 \leq t \leq T, u_{0, \min }(t) \leq x \leq u_{0, \max }(t)\right\}$ if its interior is nonempty. This question was a short time ago an open problem [20, Remark 4.4]. We note that the relation between $\mathcal{S}_{c}$ and the set $\left\{(t, x) \in \mathbb{R}^{2}: 0 \leq t \leq T, u_{c, \min }(t) \leq x \leq u_{c, \text { max }}(t)\right\}$ with $c>0$ having nonempty interior is described in Lemma $1(\mathrm{~d})$.

Theorem 1 Let $\left(\mathrm{H}_{1}\right)-\left(\mathrm{H}_{3}\right)$ hold. Let us assume that there exists $t_{0} \in(0, T)$ such that $u_{0, \min }\left(t_{0}\right)<u_{0, \max }\left(t_{0}\right)$. Then for any $Q \in\left(u_{0, \min }\left(t_{0}\right), u_{0, \max }\left(t_{0}\right)\right)$ there exists $u \in \mathcal{S}_{0}$ satisfying $u\left(t_{0}\right)=Q$.

\section{Proof Step 1. Auxiliary Dirichlet problem.}

Choose $Q \in\left(u_{0, \min }\left(t_{0}\right), u_{0, \max }\left(t_{0}\right)\right)$. Consider (3) subject to the Dirichlet conditions

$$
u\left(t_{0}\right)=Q, \quad u(T)=0 .
$$

We claim that there exists a solution $v$ to problem (3), (7) such that

$$
u_{0, \min }(t) \leq v(t) \leq u_{0, \max }(t) \quad \text { for } t \in\left[t_{0}, T\right] .
$$

We show this result utilizing the method of lower and upper functions. It follows from $\left(\mathrm{H}_{1}\right)$ that there exists $\varphi \in L^{1}\left[t_{0}, T\right]$ such that

$$
\left|\frac{a}{t^{2}} x+f(t, x)\right| \leq \varphi(t) \quad \text { for a.e. } t \in\left[t_{0}, T\right] \text { and } u_{0, \min }(t) \leq x \leq u_{0, \max }(t) .
$$


Let

$$
W=\int_{t_{0}}^{T} \varphi(t) \mathrm{d} t, \quad S=\max \left\{\left\|u_{0, \min }^{\prime}\right\|_{\infty},\left\|u_{0, \max }^{\prime}\right\|_{\infty},\left(\frac{T}{t_{0}}\right)^{|a|} W\right\}+1
$$

and let $\chi: \mathbb{R} \rightarrow[0,1]$ be given as

$$
\chi(x)= \begin{cases}1 & \text { if }|x| \leq S, \\ 2-\frac{|x|}{S} & \text { if } S<|x|<2 S \\ 0 & \text { if }|x| \geq 2 S .\end{cases}
$$

We now consider the auxiliary differential equation

$$
v^{\prime \prime}(t)=\chi\left(v^{\prime}(t)\right)\left(-\frac{a}{t} v^{\prime}(t)+\frac{a}{t^{2}} v(t)+f(t, v(t))\right)
$$

It is not difficult to verify that $\chi(y)|y| \leq S$ for $y \in \mathbb{R}$. Hence, $c f$. (9),

$$
\chi(y)\left|-\frac{a}{t} y+\frac{a}{t^{2}} x+f(t, x)\right| \leq \chi(y) \frac{|a y|}{t}+\chi(y) \varphi(t) \leq \frac{|a| S}{t}+\varphi(t)
$$

for a.e. $t \in\left[t_{0}, T\right]$ and all $u_{0, \min }(t) \leq x \leq u_{0, \max }(t), y \in \mathbb{R}$. Since $\chi\left(u_{0, \min }^{\prime}(t)\right)=1$ and $\chi\left(u_{0, \text { max }}^{\prime}(t)\right)=1$ for $t \in\left[t_{0}, T\right], u_{0, \min }(T)=u_{0, \max }(T)=0, u_{0, \min }\left(t_{0}\right)<Q<u_{0, \max }\left(t_{0}\right)$, and $u_{0, \min }, u_{0, \max }$ solve (3) on $[0, T]$, we conclude that $u_{0, \min }$ and $u_{0, \max }$ are lower and upper functions of problem (3), (7) (see e.g. [21] or [22]). This fact together with (11) implies the existence of a solution $v$ to problem (3), (7) satisfying (8), $c f$. [21, Lemma 3.7]. Moreover,

$$
\frac{u_{0, \min }(t)}{T-t} \leq \frac{v(t)}{T-t} \leq \frac{u_{0, \max }(t)}{T-t}, \quad t \in\left[t_{0}, T\right)
$$

and taking the limit $t \rightarrow T$ we obtain ${ }^{\mathrm{a}} u_{0, \min }^{\prime}(T) \geq v^{\prime}(T) \geq u_{0, \max }^{\prime}(T)$, which together with $u_{0, \text { min }}^{\prime}(T)=u_{0, \max }^{\prime}(T)=0$ gives $\nu^{\prime}(T)=0$.

We now prove that $\left|v^{\prime}\right|<S$ on $\left[t_{0}, T\right]$. The proof is indirect. Let us assume that there exists $\xi \in\left[t_{0}, T\right)$ such that $\left|v^{\prime}(\xi)\right|=S$ and $\left|v^{\prime}(t)\right|<S$ for $t \in(\xi, T]$. Then $\chi\left(v^{\prime}(t)\right)=1$ on this interval, and therefore

$$
v^{\prime \prime}(t)=-\frac{a}{t} v^{\prime}(t)+\frac{a}{t^{2}} v(t)+f(t, v(t)) \quad \text { for a.e. } t \in[\xi, T] .
$$

From (8) and (9) we conclude that

$$
\left|\frac{a}{t^{2}} v(t)+f(t, v(t))\right| \leq \varphi(t) \quad \text { for a.e. } t \in[\xi, T] .
$$

Since

$$
\begin{aligned}
-v^{\prime}(t) & =v^{\prime}(T)-v^{\prime}(t)=\int_{t}^{T} v^{\prime \prime}(s) \mathrm{d} s \\
& =-a \int_{t}^{T} \frac{v^{\prime}(s)}{s} \mathrm{~d} s+\int_{t}^{T}\left(\frac{a}{s^{2}} v(s)+f(s, v(s))\right) \mathrm{d} s,
\end{aligned}
$$


we have

$$
\left|v^{\prime}(t)\right| \leq|a| \int_{t}^{T} \frac{\left|v^{\prime}(s)\right|}{s} \mathrm{~d} s+\int_{t}^{T} \varphi(s) \mathrm{d} s .
$$

In particular,

$$
\left|v^{\prime}(t)\right| \leq|a| \int_{t}^{T} \frac{\left|v^{\prime}(s)\right|}{s} \mathrm{~d} s+W \quad \text { for } t \in[\xi, T] .
$$

By the Gronwall lemma,

$$
\left|v^{\prime}(t)\right| \leq W \exp \left(|a| \int_{t}^{T} \frac{\mathrm{d} s}{s}\right)=W\left(\frac{T}{t}\right)^{|a|}, \quad t \in[\xi, T] .
$$

Therefore

$$
S=\left|v^{\prime}(\xi)\right| \leq W\left(\frac{T}{\xi}\right)^{|a|}
$$

which contradicts

$$
W\left(\frac{T}{\xi}\right)^{|a|} \leq W\left(\frac{T}{t_{0}}\right)^{|a|} \leq S-1 .
$$

Consequently, $\left|v^{\prime}(t)\right|<S$ for $t \in\left[t_{0}, T\right]$, and so $\chi\left(v^{\prime}\right)=1$ on this interval. Thus, $v$ is a solution of problem (3), (7).

Step 2. Continuation of the solution $v$.

It follows from the arguments given in Step 1 that $v$ is a solution of problem (3), (7) on $\left[t_{0}, T\right], v^{\prime}(T)=0$ and $u_{0, \text { min }}\left(t_{0}\right)<v\left(t_{0}\right)<u_{0, \text { max }}\left(t_{0}\right)$ because $v\left(t_{0}\right)=Q \in\left(u_{0, \text { min }}\left(t_{0}\right), u_{0, \text { max }}\left(t_{0}\right)\right)$. It is easy to verify that the equality

$$
\left(t^{a+2}\left(\frac{v(t)}{t}\right)^{\prime}\right)^{\prime}=t^{a+1} f(t, v(t))
$$

is satisfied for a.e. $t \in\left[t_{0}, T\right]$. We now integrate the last equality two times over $[t, T] \subset$ $\left[t_{0}, T\right]$ and have (note that $v(T)=v^{\prime}(T)=0$ )

$$
v(t)=t \int_{t}^{T} s^{-a-2}\left(\int_{s}^{T} \xi^{a+1} f(\xi, v(\xi)) \mathrm{d} \xi\right) \mathrm{d} s, \quad t \in\left[t_{0}, T\right] .
$$

Let $u$ be a solution of problem (3), (7) on an interval $J$ which is a left-continuation of $v$. Let us assume that $u$ is not continuable. Let $\gamma=\inf \{t: t \in J\}$. Then $0 \leq \gamma<t_{0}$ and (12) with $v$ replaced by $u$ holds for a.e. $t \in J$. The integration now yields

$$
u(t)=t \int_{t}^{T} s^{-a-2}\left(\int_{s}^{T} \xi^{a+1} f(\xi, u(\xi)) \mathrm{d} \xi\right) \mathrm{d} s, \quad t \in J,
$$

and we claim that

$$
u_{0, \min }(t)<u(t)<u_{0, \max }(t) \quad \text { for } t \in\left(\gamma, t_{0}\right]
$$


We show inequality (14) indirectly. Let us assume that (14) does not hold. Then there exists $v \in\left(\gamma, t_{0}\right)$ such that

$$
u_{0, \min }(t)<u(t)<u_{0, \max }(t) \quad \text { for } t \in\left(v, t_{0}\right]
$$

and either $u(v)=u_{0, \max }(v)$ or $u(v)=u_{0, \min }(v)$. Assume that $u(v)=u_{0, \max }(v)$.

Since, by $\left(\mathrm{H}_{3}\right), f(t, u(t))<f\left(t, u_{0, \max }(t)\right)$ for a.e. $t \in\left[v, t_{0}\right]$ and $f(t, u(t)) \leq f\left(t, u_{0, \max }(t)\right)$ for a.e. $t \in\left[t_{0}, T\right]$, we have

$$
\begin{aligned}
u(v) & =v \int_{v}^{T} s^{-a-2}\left(\int_{s}^{T} \xi^{a+1} f(\xi, u(\xi)) \mathrm{d} \xi\right) \mathrm{d} s \\
& <v \int_{v}^{T} s^{-a-2}\left(\int_{s}^{T} \xi^{a+1} f\left(\xi, u_{0, \max }(\xi)\right) \mathrm{d} \xi\right) \mathrm{d} s=u_{0, \max }(v),
\end{aligned}
$$

which is not possible. The case $u(v)=u_{0, \min }(v)$ can be discussed analogously. Hence, (14) holds.

Suppose that $\gamma>0$. Then $J=(\gamma, T]$ and since $u$ is bounded on $(\gamma, T]$, it follows that $\lim \sup _{t \rightarrow \gamma^{+}}\left|u^{\prime}(t)\right|=\infty$. The integration of the equality

$$
u^{\prime \prime}(t)=-\frac{a}{t} u^{\prime}(t)+\frac{a}{t^{2}} u(t)+f(t, u(t)) \quad \text { for a.e. } t \in(\gamma, T]
$$

over $[t, T] \subset(\gamma, T]$ gives

$$
-u^{\prime}(t)=-a \int_{t}^{T} \frac{u^{\prime}(s)}{s} \mathrm{~d} s+\int_{t}^{T}\left(\frac{a}{s^{2}} u(s)+f(s, u(s))\right) \mathrm{d} s, \quad t \in(\gamma, T]
$$

Since $\left|\frac{a}{t^{2}} u(t)+f(t, u(t))\right| \leq p(t)$ for a.e. $t \in[\gamma, T]$, where $p \in L^{1}[\gamma, T]$, we have

$$
\left|u^{\prime}(t)\right| \leq|a| \int_{t}^{T} \frac{\left|u^{\prime}(s)\right|}{s} \mathrm{~d} s+\int_{\gamma}^{T} p(s) \mathrm{d} s, \quad t \in(\gamma, T] .
$$

Using the Gronwall lemma, we deduce that for $t \in(\gamma, T]$,

$$
\left|u^{\prime}(t)\right| \leq\left(\frac{T}{t}\right)^{|a|} \int_{\gamma}^{T} p(s) \mathrm{d} s \leq\left(\frac{T}{\gamma}\right)^{|a|} \int_{\gamma}^{T} p(s) \mathrm{d} s
$$

holds. This is a contradiction.

Therefore $\gamma=0$, and then (14) yields $\lim _{t \rightarrow 0^{+}} u(t)=0$. Consequently $J=[0, T]$, and the assertion of the theorem follows from (13).

In the next corollary we extend the statement (d) from Lemma 1 to a large set of $A$ values.

Corollary 1 For each $t_{0} \in(0, T)$ and each $A \geq u_{0, \min }\left(t_{0}\right)$ there exists $u \in \mathcal{S}$ satisfying $u\left(t_{0}\right)=A$.

Proof The result follows immediately from Lemma 1(d) and Theorem 1. 
Remark 1 Corollary 1 says that the set $\mathcal{U}=\left\{(t, x) \in \mathbb{R}^{2}: t \in[0, T], x \geq u_{0, \min }(t)\right\}$ is covered by the graphs of functions from $\mathcal{S}$, that is,

$$
\mathcal{U}=\{(t, u(t)): t \in[0, T], u \in \mathcal{S}\} .
$$

By Lemma 1(b), (c) we know that functions from $\mathcal{S}$ are uniquely determined by the values $-c$ of their derivatives at the right end point $t=T$ only in the case that $\mathcal{S}_{c}$ is a singleton set for each $c \geq 0$. Since we cannot uniquely determine all functions from $\mathcal{S}$ via their derivatives at $t=T$, see Lemma 1(e), we discuss their derivatives at the singular point $t=0$.

Lemma 2 Let $\left(\mathrm{H}_{1}\right)-\left(\mathrm{H}_{3}\right)$ hold. Let $u \in \mathcal{S}_{c}, c \geq 0$. Then

$$
u^{\prime}(0)=\frac{c}{|a+1|}+\int_{0}^{T} s^{-a-2}\left(\int_{s}^{T} \xi^{a+1} f(\xi, u(\xi)) \mathrm{d} \xi\right) \mathrm{d} s .
$$

Proof It follows from Lemma 1 (g) that (6) holds for $t \in[0, T]$. Since $u(0)=0$, we have (note that $a+1<0)$

$$
\begin{aligned}
u^{\prime}(0)= & \lim _{t \rightarrow 0+} \frac{u(t)-u(0)}{t} \\
= & \lim _{t \rightarrow 0+}\left(\frac{c T^{a+1}}{|a+1|}\left(T^{-a-1}-t^{-a-1}\right)\right. \\
& \left.+\int_{t}^{T} s^{-a-2}\left(\int_{s}^{T} \xi^{a+1} f(\xi, u(\xi)) \mathrm{d} \xi\right) \mathrm{d} s\right) \\
= & \frac{c}{|a+1|}+\int_{0}^{T} s^{-a-2}\left(\int_{s}^{T} \xi^{a+1} f(\xi, u(\xi)) \mathrm{d} \xi\right) \mathrm{d} s,
\end{aligned}
$$

and (15) follows.

Corollary 2 Let $u \in \mathcal{S}$. Then

$$
u^{\prime}(0)=-\frac{u^{\prime}(T)}{|a+1|}+\int_{0}^{T} s^{-a-2}\left(\int_{s}^{T} \xi^{a+1} f(\xi, u(\xi)) \mathrm{d} \xi\right) \mathrm{d} s .
$$

Proof The result follows from Lemma 2, since $u \in \mathcal{S}_{-u^{\prime}(T)}$.

Corollary 3 Let $u, v \in \mathcal{S}, u \neq v$. Then $u^{\prime}(0) \neq v^{\prime}(0)$.

Proof Since $u \neq v$, there exists $t_{0} \in(0, T)$ such that $u\left(t_{0}\right) \neq v\left(t_{0}\right)$. We can assume that for instance $u\left(t_{0}\right)<v\left(t_{0}\right)$. Then $u<v$ on $\left(0, t_{0}\right]$ and $u \leq v$ on $\left(t_{0}, T\right]$ by Lemma $1(\mathrm{~b})(\mathrm{c})$. Hence, $u^{\prime}(T) \geq v^{\prime}(T)$ and

$$
\begin{aligned}
& \int_{0}^{T} s^{-a-2}\left(\int_{s}^{T} \xi^{a+1} f(\xi, u(\xi)) \mathrm{d} \xi\right) \mathrm{d} s \\
& \quad<\int_{0}^{T} s^{-a-2}\left(\int_{s}^{T} \xi^{a+1} f(\xi, v(\xi)) \mathrm{d} \xi\right) \mathrm{d} s,
\end{aligned}
$$

which together with Corollary 2 gives $u^{\prime}(0)<v^{\prime}(0)$. 
Corollary 4 Let $u \in \mathcal{S}_{c}, c \geq 0$. Then

$$
u^{\prime}(0)>\frac{c}{|a+1|}
$$

In particular, $u_{0, \min }^{\prime}(0)>0$.

Proof Inequality (16) follows from Lemma 2 and the fact that $f(t, u(t))>0$ for a.e. $t \in[0, T]$ by $\left(\mathrm{H}_{2}\right)$.

Corollary 5 Let $u \in \mathcal{S}$. Then

$$
\int_{0}^{T} s^{-a-2}\left(\int_{s}^{T} \xi^{a+1} f(\xi, u(\xi)) \mathrm{d} \xi\right) \mathrm{d} s=\frac{1}{|a+1|} \int_{0}^{T} f(s, u(s)) \mathrm{d} s .
$$

Proof The integration of (3) over $[\varepsilon, T] \subset(0, T]$ gives

$$
u^{\prime}(T)-u^{\prime}(\varepsilon)+a \int_{\varepsilon}^{T} \frac{u^{\prime}(t)}{t} \mathrm{~d} t-a \int_{\varepsilon}^{T} \frac{u(t)}{t^{2}} \mathrm{~d} t=\int_{\varepsilon}^{T} f(t, u(t)) \mathrm{d} t .
$$

Using integration by parts we obtain

$$
\int_{\varepsilon}^{T} \frac{u(t)}{t^{2}} \mathrm{~d} t=\frac{u(\varepsilon)}{\varepsilon}+\int_{\varepsilon}^{T} \frac{u^{\prime}(t)}{t} \mathrm{~d} t
$$

and, therefore,

$$
u^{\prime}(T)-u^{\prime}(\varepsilon)-a \frac{u(\varepsilon)}{\varepsilon}=\int_{\varepsilon}^{T} f(t, u(t)) \mathrm{d} t
$$

Taking the limit $\varepsilon \rightarrow 0$, we have

$$
u^{\prime}(T)+|a+1| u^{\prime}(0)=\int_{0}^{T} f(t, u(t)) \mathrm{d} t
$$

On the other hand, it follows from Corollary 2 that

$$
u^{\prime}(T)+|a+1| u^{\prime}(0)=|a+1| \int_{0}^{T} s^{-a-2}\left(\int_{s}^{T} \xi^{a+1} f(\xi, u(\xi)) \mathrm{d} \xi\right) \mathrm{d} s
$$

Combining the above two equalities yields the result.

Lemma 3 Let $\left(\mathrm{H}_{1}\right)-\left(\mathrm{H}_{3}\right)$ hold. Let $c \geq 0$ and $\mathcal{S}_{c}$ be not a singleton set. Then for each $\alpha \in\left[u_{c, \min }^{\prime}(0), u_{c, \max }^{\prime}(0)\right]$ there exists a unique $u \in \mathcal{S}_{c}$ such that $u^{\prime}(0)=\alpha$. Consequently, $\left[u_{c, \min }^{\prime}(0), u_{c, \max }^{\prime}(0)\right]=\left\{u^{\prime}(0): u \in \mathcal{S}_{c}\right\}$. 
Proof Let $u \in \mathcal{S}_{c}, u_{c \text {, min }} \neq u \neq u_{c \text {, max }}$. Then it follows from Lemma 1 (a) and $\left(\mathrm{H}_{3}\right)$ that

$$
\begin{gathered}
\int_{0}^{T} s^{-a-2}\left(\int_{s}^{T} \xi^{a+1} f\left(\xi, u_{c, \min }(\xi)\right) \mathrm{d} \xi\right) \mathrm{d} s \\
\quad<\int_{0}^{T} s^{-a-2}\left(\int_{s}^{T} \xi^{a+1} f(\xi, u(\xi)) \mathrm{d} \xi\right) \mathrm{d} s \\
<\int_{0}^{T} s^{-a-2}\left(\int_{s}^{T} \xi^{a+1} f\left(\xi, u_{c, \max }(\xi)\right) \mathrm{d} \xi\right) \mathrm{d} s
\end{gathered}
$$

Hence, by Lemma 2,

$$
u_{c, \text { min }}^{\prime}(0)<u^{\prime}(0)<u_{c, \text { max }}^{\prime}(0) \text { for } u \in \mathcal{S}_{c}, u_{c, \min } \neq u \neq u_{c, \max } \text {. }
$$

Since $\mathcal{S}_{c}$ is a compact set in $C^{1}[0, T]$ by Lemma $1(\mathrm{f})$, the set $I=\left\{u^{\prime}(0): u \in \mathcal{S}_{c}\right\} \subset$ $\left[u_{c \text {,min }}^{\prime}(0), u_{c \text {, max }}^{\prime}(0)\right]$ is closed. In fact, let $\left\{\beta_{n}\right\} \subset I, \beta_{n}=u_{n}^{\prime}(0)$, where $u_{n} \in \mathcal{S}_{c}$, and let $\lim _{n \rightarrow \infty} \beta_{n}=\beta$. Then there exist a subsequence $\left\{\beta_{k_{n}}\right\}$ of $\left\{\beta_{n}\right\}$ and $u \in \mathcal{S}_{c}$ such that $\lim _{n \rightarrow \infty} u_{k_{n}}=u$ in $C^{1}[0, T]$. In particular, $\beta_{k_{n}}=u_{k_{n}}^{\prime}(0) \rightarrow u^{\prime}(0)$ as $n \rightarrow \infty$. Therefore, $\beta=u^{\prime}(0) \in I$.

It remains to prove that $I=\left[u_{c, \text { min }}^{\prime}(0), u_{c \text {, max }}^{\prime}(0)\right]$. Assume that the equality does not hold. Then, from the structure of bounded and closed subsets of $\mathbb{R}$ the existence of an open interval $(\alpha, \beta) \subset\left[u_{c \text {,min }}^{\prime}(0), u_{c \text {,max }}^{\prime}(0)\right] \backslash I, \alpha, \beta \in I$, follows. Let $\alpha=u_{\alpha}^{\prime}(0)$ and $\beta=u_{\beta}^{\prime}(0)$, where $u_{\alpha}, u_{\beta} \in \mathcal{S}_{c}$. Due to Lemma $1(\mathrm{c})$, there exists $t_{0} \in(0, T)$ such that $u_{\alpha}<u_{\beta}$ on $\left(0, t_{0}\right], u_{\alpha} \leq u_{\beta}$ on $\left(t_{0}, T\right]$. Choose $\tau \in\left(u_{\alpha}\left(t_{0}\right), u_{\beta}\left(t_{0}\right)\right)$. By Corollary 1 , there exists $v \in \mathcal{S}_{c}$ such that $v\left(t_{0}\right)=\tau$. Then $u_{\alpha}<v<u_{\beta}$ on $\left(0, t_{0}\right]$ and $u_{\alpha} \leq v \leq u_{\beta}$ on $\left(t_{0}, T\right]$. Consequently, $u_{\alpha}^{\prime}(0)<v^{\prime}(0)<u_{\beta}^{\prime}(0)$ on $\left(t_{0}, T\right]$, that is, $v^{\prime}(0) \in(\alpha, \beta)$, which is not possible.

Since functions from $\mathcal{S}$ belong to $A C^{1}[0, T]$, we have $u^{\prime}(0)<\infty$ for each $u \in \mathcal{S}$. Corollary 4 yields $u_{0, \text { min }}^{\prime}(0)>0$. Let us denote

$$
J:=\left[u_{0, \min }^{\prime}(0), \infty\right) \subset(0, \infty)
$$

Lemma 3 implies that functions from $\mathcal{S}$ can be uniquely determined by the values of their derivatives at the singular point $t=0$; see Theorem 2 .

Theorem 2 Let $\left(\mathrm{H}_{1}\right)-\left(\mathrm{H}_{3}\right)$ hold. Then there exists a unique $u \in \mathcal{S}$ satisfying $u^{\prime}(0)=\alpha$ if and only if $\alpha \in J$.

Proof We first show

$$
\left\{u^{\prime}(0): u \in \mathcal{S}\right\}=J
$$

It follows from Lemma 3 that

$$
\left\{u^{\prime}(0): u \in \bigcup_{0 \leq c \leq K} \mathcal{S}_{c}\right\}=\left[u_{0, \min }^{\prime}(0), u_{K, \max }^{\prime}(0)\right]
$$

for each $K \geq 0$, where we set $u_{c \text {, min }}=u=u_{c \text {, max }}$ if $\mathcal{S}_{c}$ is a singleton set and $\mathcal{S}_{c}=\{u\}$. In view of Corollary $4, u^{\prime}(0)>c /|a+1|$ for $u \in \mathcal{S}_{c}$. Consequently, (18) follows. 
Let us now choose $\alpha \in J$. Then there exists $u \in \mathcal{S}$ satisfying $u^{\prime}(0)=\alpha$. The uniqueness of $u$ follows from Corollary 3.

For $\alpha \in J$, we denote by $u_{\alpha}$ the unique element of $\mathcal{S}$ satisfying $u_{\alpha}^{\prime}(0)=\alpha$.

Theorem 3 Let $\left(\mathrm{H}_{1}\right)-\left(\mathrm{H}_{3}\right)$ hold. Assume that $\left\{\alpha_{n}\right\} \subset J$ is a convergent sequence and $\lim _{n \rightarrow \infty} \alpha_{n}=\alpha$. Then $\lim _{n \rightarrow \infty} u_{\alpha_{n}}=u_{\alpha}$ in $C^{1}[0, T]$.

Proof Let $K=\sup \left\{-u_{\alpha_{n}}^{\prime}(T): n \in \mathbb{N}\right\}$. Then $K<\infty$ because $\alpha_{n}=u_{\alpha_{n}}^{\prime}(0)>-u_{\alpha_{n}}^{\prime}(T) /|a+1|$ for $n \in \mathbb{N}$ by Corollary 4 . Since $\left\{u_{\alpha_{n}}\right\} \subset \bigcup_{0 \leq c \leq K} \mathcal{S}_{c}$ and $\bigcup_{0 \leq c \leq K} \mathcal{S}_{c}$ is compact in $C^{1}[0, T]$ by Lemma $1(\mathrm{f})$, the sequence $\left\{u_{\alpha_{n}}\right\}$ is relatively compact in $C^{1}[0, T]$. Let $\left\{u_{\alpha_{\ell_{n}}}\right\}$ be a subsequence of $\left\{u_{\alpha_{n}}\right\}$ which is convergent in $C^{1}[0, T]$, and $\operatorname{let}_{\lim } \rightarrow \infty u_{\alpha_{\ell_{n}}}=u$. Then $u \in \mathcal{S}_{c_{0}}$ for a $c_{0} \in[0, K]$ and $u^{\prime}(0)=\lim _{n \rightarrow \infty} u_{\alpha_{\ell_{n}}}^{\prime}(0)=\lim _{n \rightarrow \infty} \alpha_{\ell_{n}}=\alpha$. Therefore, $u=u_{\alpha}$ and hence any subsequence $\left\{u_{\alpha_{\ell_{n}}}\right\}$ of $\left\{u_{\alpha_{n}}\right\}$ converging in $C^{1}[0, T]$ has the same limit $u_{\alpha}$. Consequently, $\left\{u_{\alpha_{n}}\right\}$ is convergent in $C^{1}[0, T]$ and $u_{\alpha}$ is its limit.

\section{Blow-up solutions and their properties}

This section contains the main results of the paper. First, we present a lemma which describes how positive solutions of (1) may behave at the singular point $t=0$.

Lemma 4 Let $k \in(1, \infty)$ and let the function $h \in \operatorname{Car}([0, T] \times(0, \infty))$ be positive. Let $v$ be a positive solution of (1). Then either

$$
\lim _{t \rightarrow 0+} v(t) \in[0, \infty), \quad \lim _{t \rightarrow 0+} v^{\prime}(t)=0,
$$

or

$$
\lim _{t \rightarrow 0+} v(t)=\infty, \quad \lim _{t \rightarrow 0+} v^{\prime}(t)=-\infty
$$

Proof By Corollary 3.5 in [12], if $\sup \left\{|v(t)|+\left|v^{\prime}(t)\right|: t \in(0, T]\right\}<\infty$, then $v$ satisfies (19). Now, assume that

$$
\sup \left\{|v(t)|+\left|v^{\prime}(t)\right|: t \in(0, T]\right\}=\infty .
$$

Then $v$ has to satisfy

$$
\sup \left\{\left|v^{\prime}(t)\right|: t \in(0, T]\right\}=\infty
$$

because otherwise, if (22) was not true, then $\sup \{|v(t)|: t \in(0, T]\}<\infty$, contradicting (21). Since $h$ is positive, (1) indicates that the function $t^{k} v^{\prime}(t)$ is increasing on $(0, T]$ and hence there exists $\lim _{t \rightarrow 0+} t^{k} v^{\prime}(t)$. The next part of the proof is divided into three cases.

(i) Let us assume that

$$
\lim _{t \rightarrow 0+} t^{k} v^{\prime}(t)=0
$$

Since $t^{k} v^{\prime}(t)$ is increasing on $(0, T]$, we deduce $t^{k} v^{\prime}(t)>0$ and $v^{\prime}(t)>0$ for $t \in(0, T]$. Consequently, $v$ is increasing on $(0, T]$. This together with $v>0$ and $v \in A C_{\text {loc }}^{1}(0, T]$ yields 
$\sup \{v(t): t \in(0, T]\}<\infty$. Therefore, $h(t, v(t)) \in L^{1}[0, T]$. Integrating (1) and using (23), we obtain

$$
\begin{aligned}
& t^{k} v^{\prime}(t)=\int_{0}^{t} s^{k} h(s, v(s)) \mathrm{d} s, \quad t \in(0, T], \\
& 0<v^{\prime}(t)=\frac{1}{t^{k}} \int_{0}^{t} s^{k} h(s, v(s)) \mathrm{d} s<\int_{0}^{T} h(s, v(s)) \mathrm{d} s<\infty, \quad t \in(0, T],
\end{aligned}
$$

which is a contradiction to (22).

(ii) Assume that

$$
\lim _{t \rightarrow 0+} t^{k} v^{\prime}(t)=\ell>0 .
$$

Then there exists $t_{0} \in(0, T)$ such that

$$
v^{\prime}(t)>\ell / 2 t^{k} \text { for } t \in\left(0, t_{0}\right] .
$$

Hence, $v$ is increasing on $\left(0, t_{0}\right]$ and there exists $\lim _{t \rightarrow 0+} v(t) \geq 0$.

By integration, we obtain from (24)

$$
v\left(t_{0}\right)-v(t)>\frac{\ell\left(t_{0}^{1-k}-t^{1-k}\right)}{2(1-k)}, \quad t \in\left(0, t_{0}\right]
$$

and, by virtue of $k>1$ and $\ell>0$, we arrive at

$$
\lim _{t \rightarrow 0+} v(t) \leq v\left(t_{0}\right)-\lim _{t \rightarrow 0+} \frac{\ell\left(t_{0}^{1-k}-t^{1-k}\right)}{2(1-k)}=-\infty,
$$

which again is a contradiction.

(iii) Assume that

$$
\lim _{t \rightarrow 0+} t^{k} v^{\prime}(t)=\ell<0 .
$$

Then there exists $t_{0} \in(0, T)$ such that

$$
\nu^{\prime}(t)<\ell / 2 t^{k} \text { for } t \in\left(0, t_{0}\right] .
$$

Hence, $v$ is decreasing on $\left(0, t_{0}\right]$ and there exists $\lim _{t \rightarrow 0+} v(t) \in(0, \infty]$. By integration, we obtain from (26)

$$
v\left(t_{0}\right)-v(t)<\frac{\ell\left(t_{0}^{1-k}-t^{1-k}\right)}{2(1-k)}, \quad t \in\left(0, t_{0}\right]
$$

and, since $k>1$ and $\ell<0$, we have

$$
\lim _{t \rightarrow 0+} v(t) \geq v\left(t_{0}\right)-\lim _{t \rightarrow 0+} \frac{\ell\left(t_{0}^{1-k}-t^{1-k}\right)}{2(1-k)}=\infty .
$$

In addition (25) yields $\lim _{t \rightarrow 0+} \nu^{\prime}(t)=-\infty$ and (20) follows. 
Now, we investigate the existence and properties of blow-up solutions of (1), for the case that the function $h$ has the form

$$
h(t, x)=\psi(t)+g(t, x) .
$$

In particular, we study the equation

$$
v^{\prime \prime}(t)+\frac{k}{t} v^{\prime}(t)=\psi(t)+g(t, v(t)),
$$

where $k \in(1, \infty)$ and $\psi, g$ satisfy the conditions

$\left(\mathrm{H}_{1}^{0}\right) \psi \in L^{1}[0, T]$ and $\psi>0$ for a.e. $t \in[0, T]$,

$\left(\mathrm{H}_{2}^{0}\right) g \in \operatorname{Car}([0, T] \times[0, \infty))$ and $g(t, x)$ is increasing in $x$ for a.e. $t \in[0, T]$,

$\left(\mathrm{H}_{3}^{0}\right) \quad 0 \leq g(t, x) \leq \phi(x)$ for a.e. $t \in[0, T]$ and all $x \in[0, \infty)$, where $\phi \in C[0, \infty)$, $\phi$ is increasing and

$$
\lim _{x \rightarrow \infty} \frac{\phi(x)}{x}=0 .
$$

Together with (27) we consider the conditions

$$
\lim _{t \rightarrow 0} v(t)=\infty, \quad v(T)=0,
$$

and

$$
v^{\prime}(T)=-c .
$$

We define a positive solution to problem (27), (28) as a function $v \in A C_{\mathrm{loc}}^{1}(0, T]$ such that $v>0$ on $(0, T), v$ satisfies the boundary conditions (28), and (27) holds for a.e. $t \in[0, T]$.

Define a set $\mathcal{Z}$ by

$$
\mathcal{Z}=\left\{v \in A C_{\mathrm{loc}}^{1}(0, T]: v \text { is a positive solution of }(27),(28)\right\}
$$

Clearly, for each $v \in \mathcal{Z}$ there exists $c \geq 0$ such that (29) holds.

Lemma 5 Let $\left(\mathrm{H}_{1}^{0}\right)-\left(\mathrm{H}_{3}^{0}\right)$ hold. Let $v \in \mathcal{Z}$. Then $v^{\prime}<0$ on $(0, T)$.

Proof Since $\left(t^{k} v^{\prime}\right)^{\prime}=t^{k}\left(v^{\prime \prime}+(k / t) v^{\prime}\right)$, the integration of (27) over the subinterval $[t, T] \subset$ $(0, T]$ gives

$$
T^{k} v^{\prime}(T)-t^{k} v^{\prime}(t)=\int_{t}^{T} s^{k}(\psi(s)+g(s, v(s))) \mathrm{d} s .
$$

Assume that $v^{\prime}\left(t_{0}\right)=0$ for some $t_{0} \in(0, T)$. Then

$$
T^{k} v^{\prime}(T)=\int_{t_{0}}^{T} s^{k}(\psi(s)+g(s, v(s))) \mathrm{d} s,
$$

which is not possible, since $\nu^{\prime}(T) \leq 0$ and $\int_{t_{0}}^{T} s^{k}(\psi(s)+g(s, v(s))) \mathrm{d} s>0$. 
Moreover, let us define sets

$$
\mathcal{R}=\left\{v \in \mathcal{Z}: t^{k} v \text { is bounded on }(0, T]\right\}
$$

and

$$
\mathcal{R}_{c}=\left\{v \in \mathcal{R}: v^{\prime}(T)=-c\right\} \quad \text { for } c \geq 0 .
$$

It is obvious that $\mathcal{R}=\bigcup_{c \geq 0} \mathcal{R}_{c}$. Using the substitution

$$
a=-k, \quad u(t)=t^{-a} v(t)=t^{k} v(t) \quad \text { for } t \in(0, T],
$$

we can rewrite (27) and obtain the form

$$
u^{\prime \prime}(t)+\frac{a}{t} u^{\prime}(t)-\frac{a}{t^{2}} u(t)=t^{-a}\left(\psi(t)+g\left(t, t^{a} x\right)\right) .
$$

We now introduce a function $f$,

$$
f(t, x)=t^{-a}\left(\psi(t)+g\left(t, t^{a} x\right)\right) \quad \text { for a.e. } t \in[0, T] \text { and all } x \in[0, \infty) .
$$

Under conditions $\left(\mathrm{H}_{1}^{0}\right)-\left(\mathrm{H}_{3}^{0}\right)$, the function $f$ satisfies $\left(\mathrm{H}_{1}\right)-\left(\mathrm{H}_{3}\right)$; see the proof of $[20$, Theorem 5.1]. Therefore, the results of Section 2 hold for problem (34), (4). As in Section 2, we define the sets $\mathcal{S}$ and $\mathcal{S}_{c}$ for solutions of (34).

The following result is the key-stone to the analysis of the structure of the set $\mathcal{R}$ and describes the relation between the sets $\mathcal{S}$ and $\mathcal{R}$.

Theorem 4 Let $\left(\mathrm{H}_{1}^{0}\right)-\left(\mathrm{H}_{3}^{0}\right)$ hold and $c \geq 0$. Let us assume that (33) holds. Then $v \in \mathcal{R}_{c}$ if and only if $u \in \mathcal{S}_{c_{0}}$ and $c_{0}=T^{k} c$.

Proof $\left(\Rightarrow\right.$ ) Let $v \in \mathcal{R}_{c}$. Then (28), (32), and (33) provide the following properties: $u \in$ $A C_{\text {loc }}^{1}(0, T], u^{\prime}(T)=-c_{0}=-T^{k} c$, and $u$ is bounded on $(0, T]$. It follows from the equality

$$
\left(t^{k} v^{\prime}(t)\right)^{\prime}=t^{k}(\psi(t)+g(t, v(t))) \text { for a.e. } t \in[0, T]
$$

that

$$
\left(t^{-a}\left(t^{a} u(t)\right)^{\prime}\right)^{\prime}=t^{-a}\left(\psi(t)+g\left(t, t^{a} u(t)\right)\right)=f(t, u(t)) \text { for a.e. } t \in[0, T] .
$$

Hence,

$$
u^{\prime \prime}(t)+\frac{a}{t} u^{\prime}(t)-\frac{a}{t^{2}} u(t)=f(t, u(t)) \quad \text { for a.e. } t \in[0, T]
$$

We now argue as in the proof of [20, Lemma 3.3] and arrive at

$$
u(t)=t \frac{c_{0} T^{a+1}}{|a+1|}\left(T^{-a-1}-t^{-a-1}\right)+t \int_{t}^{T} s^{-a-2}\left(\int_{s}^{T} \xi^{a+1} f(\xi, u(\xi)) \mathrm{d} \xi\right) \mathrm{d} s
$$


for $t \in(0, T]$. Since $u$ is bounded on $(0, T]$, we have $f(t, u(t)) \in L^{1}[0, T]$. Then, by [20, Lemma 2.1],

$$
\begin{aligned}
& 0 \leq t^{-a-1} \int_{t}^{T} s^{a+1} f(s, u(s)) \mathrm{d} s \leq \int_{0}^{T} f(s, u(s)) \mathrm{d} s \\
& 0 \leq \int_{t}^{T} s^{-a-2}\left(\int_{s}^{T} \xi^{a+1} f(\xi, u(\xi)) \mathrm{d} \xi\right) \mathrm{d} s \leq \frac{1}{|a+1|} \int_{0}^{T} f(s, u(s)) \mathrm{d} s,
\end{aligned}
$$

for $t \in(0, T]$, and hence, $\sup \left\{|u(t)|+\left|u^{\prime}(t)\right|: t \in(0, T]\right\}<\infty$. Now, [23, Corollary 1] guarantees that $u$ can be extended on $[0, T]$ with $u \in A C^{1}[0, T]$ such that the equality (35) holds for $t \in[0, T]$. Consequently, $u \in \mathcal{S}_{c_{0}}$.

$(\Leftarrow)$ Let $u \in \mathcal{S}_{c_{0}}$ and $c_{0}=T^{k} c$. Then it follows from (4), (5), and (33) that $v \in A C_{\mathrm{loc}}^{1}(0, T]$ and $v^{\prime}(T)=-c, \lim _{t \rightarrow 0+} t^{k} v(t)=u(0)=0$. Hence, $t^{k} v$ is bounded on $(0, T]$ and

$$
\left(t^{-a}\left(t^{a} u(t)\right)^{\prime}\right)^{\prime}=f(t, u(t)) \quad \text { for a.e. } t \in[0, T]
$$

yields $\left(t^{k} v^{\prime}(t)\right)^{\prime}=f\left(t, t^{k} v(t)\right)$, that is,

$$
v^{\prime \prime}(t)+\frac{k}{t} v(t)=t^{-k} f\left(t, t^{k} v(t)\right)=\psi(t)+g(t, v(t)) \quad \text { for a.e. } t \in[0, T] .
$$

Since $u \in \mathcal{S}_{c_{0}}$, the equality (35) holds on $[0, T]$, and consequently,

$$
t^{a} u(t) \geq t^{a+1} \int_{t}^{T} s^{-a-2}\left(\int_{s}^{T} \xi^{a+1} f(\xi, u(\xi)) \mathrm{d} \xi\right) \mathrm{d} s, \quad t \in(0, T]
$$

Hence, note that $a+1<0, \lim _{t \rightarrow 0} t^{a} u(t)=\infty$, and so $\lim _{t \rightarrow 0} v(t)=\infty$. As a result, we have $v \in \mathcal{R}_{c}$.

Now, we are in the position to provide results on the solvability of problem (27), (28) and formulate the properties of its solutions.

Theorem 5 Let $\left(\mathrm{H}_{1}^{0}\right)-\left(\mathrm{H}_{3}^{0}\right)$ hold. Then the following statements hold:

(a) For each $c \geq 0$ the set $\mathcal{R}_{c}$ is nonempty and there exist $v_{c, \min }, v_{c, \max } \in \mathcal{R}_{c}$ such that $v_{c, \min }(t) \leq v(t) \leq v_{c, \max }(t)$ for $t \in(0, T]$ and $v \in \mathcal{R}_{c}$.

(b) If $c_{1}>c_{2} \geq 0, v_{i} \in \mathcal{R}_{c_{i}}, i=1,2$, then $v_{1}(t)>v_{2}(t)$ for $t \in(0, T)$.

(c) If $c \geq 0, v_{i} \in \mathcal{R}_{c}, i=1,2$, and $v_{1}\left(t_{0}\right)>v_{2}\left(t_{0}\right)$ for some $t_{0} \in(0, T)$, then either $v_{1}(t)>v_{2}(t)$ for $t \in(0, T)$ or there exists $t_{*} \in\left(t_{0}, T\right)$ such that $v_{1}(t)>v_{2}(t)$ for $t \in\left(0, t_{*}\right)$ and $v_{1}(t)=v_{2}(t)$ for $t \in\left[t_{*}, T\right]$.

(d) $\mathcal{R}_{c}$ is a singleton set for each $c \in[0, \infty) \backslash \Gamma$, where $\Gamma \subset[0, \infty)$ is at most countable.

Proof The result follows by combining results from Lemma 1(a), (b), (c), and (e) with those from Theorem 4.

If $c \geq 0, c_{0}=T^{k} c$, and if (33) holds, then Theorems 4 and 5 yield

$$
v_{c, \text { min }}(t)=t^{-k} u_{c_{0}, \min }(t), \quad v_{c, \max }(t)=t^{-k} u_{c_{0}, \max }(t), \quad t \in(0, T] .
$$


The next theorem shows that the set

$$
\mathcal{V}=\left\{(t, x) \in \mathbb{R}^{2}: t \in(0, T], x \geq v_{0, \min }(t)\right\}
$$

is covered by graphs of the functions from $\mathcal{R}$.

Theorem 6 Let $\left(\mathrm{H}_{1}^{0}\right)-\left(\mathrm{H}_{3}^{0}\right)$ hold. Then, for each $t_{0} \in(0, T)$ and each $Q \geq v_{0, \min }\left(t_{0}\right)$, there exists $v \in \mathcal{R}$ satisfying $v\left(t_{0}\right)=Q$. In particular, if for some $c \geq 0$ and $\tilde{t} \in(0, T)$ the inequality $v_{c, \text { min }}(\tilde{t})<v_{c, \max }(\tilde{t})$ holds, then for each $\tilde{Q} \in\left(v_{c, \min }(\tilde{t}), v_{c, \max }(\tilde{t})\right)$ there exist $v \in \mathcal{R}_{c}$ satisfying $v(\tilde{t})=\tilde{Q}$.

Proof Choose $t_{0} \in(0, T)$ and $Q \geq v_{0, \min }\left(t_{0}\right)$. Define $A:=t_{0}^{k} Q$. Then, using (33) and (36), we deduce $u_{0, \min }\left(t_{0}\right)=t_{0}^{k} v_{0, \min }\left(t_{0}\right) \geq A$. Therefore, by Corollary 1 , there exists $u \in \mathcal{S}$ such that $u\left(t_{0}\right)=A$. Consequently, (33) and Theorem 4 give $v \in \mathcal{R}$ and $v\left(t_{0}\right)=Q$. The last statement follows from Theorem $5(\mathrm{c})$.

By Corollary 4, there exists $\alpha_{0}>0$ such that

$$
\alpha_{0}=u_{0, \min }^{\prime}(0)=\lim _{t \rightarrow 0+} \frac{u_{0, \min }(t)}{t},
$$

and hence, by (33) and (36) with $c=c_{0}=0$,

$$
\alpha_{0}=\lim _{t \rightarrow 0+} t^{k-1} v_{0, \min }(t)
$$

Using constants from the interval $\left[\alpha_{0}, \infty\right)$, we can uniquely determine all functions in $\mathcal{R}$.

Theorem 7 Let $\left(\mathrm{H}_{1}^{0}\right)-\left(\mathrm{H}_{3}^{0}\right)$ hold and let $\alpha_{0}$ be as in (38). Then the following statements hold:

(a) For each $v \in \mathcal{R}$ there exists $\alpha \in\left[\alpha_{0}, \infty\right)$ such that

$$
\lim _{t \rightarrow 0+} t^{k-1} v(t)=\alpha, \quad \lim _{t \rightarrow 0+} t^{k} v^{\prime}(t)=(1-k) \alpha .
$$

(b) For each $\alpha \in\left[\alpha_{0}, \infty\right)$ there exists a unique $v \in \mathcal{R}$ satisfying (39).

Proof (a) Choose $v \in \mathcal{R}$. Using (33), (37), and Theorem 4, we have $t^{k} v=u \in \mathcal{S}$ and

$$
\lim _{t \rightarrow 0+} t^{k-1} v(t)=\lim _{t \rightarrow 0} \frac{u(t)}{t}=u^{\prime}(0) .
$$

By Lemma $1(\mathrm{c}), u^{\prime}(0) \geq \alpha_{0}$. Hence, if we denote $u^{\prime}(0)=\alpha$, the first condition in (39) follows. Moreover,

$$
\lim _{t \rightarrow 0+} t^{k} v^{\prime}(t)=\lim _{t \rightarrow 0+} t^{k}\left(t^{-k} u(t)\right)^{\prime}=\lim _{t \rightarrow 0+}\left(-k \frac{u(t)}{t}+u^{\prime}(t)\right)=(1-k) u^{\prime}(0),
$$

and therefore the second condition in (39) holds.

(b) Choose $\alpha \in\left[\alpha_{0}, \infty\right)$. Theorem 2 guarantees that there exists a unique $u \in \mathcal{S}$ satisfying $u^{\prime}(0)=\alpha$. Using (33) and Theorem 4, we conclude $t^{-k} u=v \in \mathcal{R}$. Then $v$ satisfies (40) 
and (41) which results in (39). It remains to prove that $v$ is unique. Assume that there exists a function $w \in \mathcal{R}, w \neq v$, such that $w$ satisfies (39). Let $z=t^{k} w$. Then $z \in \mathcal{S}$ and $z^{\prime}(0)=\lim _{t \rightarrow 0} z(t) / t=\lim _{t \rightarrow 0} t^{k-1} w(t)=\alpha$. Consequently, by Theorem 2 , we arrive at $z=u$ and $w=v$, which is a contradiction.

Let $\alpha_{0}$ be given in (38) and choose $\alpha \in\left[\alpha_{0}, \infty\right)$. Keeping Theorem 7(b) in mind, there exists a unique $v \in \mathcal{R}$ satisfying (39). We denote such $v$ by $v_{\alpha}$ and define a function $w_{\alpha}$ : $[0, T] \rightarrow \mathbb{R}$ as

$$
w_{\alpha}(t):= \begin{cases}t^{k-1} v_{\alpha}(t) & \text { if } t \in(0, T] \\ \alpha & \text { if } t=0 .\end{cases}
$$

Then $w_{\alpha} \in C[0, T]$. We now specify further properties of functions $w_{\alpha}$.

Theorem 8 Let $\left(\mathrm{H}_{1}^{0}\right)-\left(\mathrm{H}_{3}^{0}\right)$ hold and let $\alpha_{0}$ be from (38). Let $\alpha, \beta \in\left[\alpha_{0}, \infty\right)$ and $\alpha<\beta$. Then either $w_{\alpha}(t)<w_{\beta}(t)$ for $t \in[0, T)$ or there exists $t_{*} \in(0, T)$ such that $w_{\alpha}(t)<w_{\beta}(t)$ for $t \in\left[0, t_{*}\right)$ and $w_{\alpha}(t)=w_{\beta}(t)$ for $t \in\left[t_{*}, T\right]$.

Proof According to (42), there exist $c_{1}, c_{2} \in[0, \infty), v_{\alpha} \in \mathcal{R}_{c_{1}}$, and $v_{\beta} \in \mathcal{R}_{c_{2}}$ such that $w_{\alpha}(t)=t^{k-1} v_{\alpha}(t), w_{\beta}(t)=t^{k-1} v_{\beta}(t)$ for $t \in(0, T]$. Also, $w_{\alpha}, w_{\beta} \in C[0, T]$ and, since $w_{\alpha}(0)=$ $\alpha<\beta=w_{\beta}(0)$, it follows that $w_{\alpha}<w_{\beta}$ in a right neighborhood of $t=0$. Therefore, there exists $t_{0} \in(0, T)$ such that $v_{\alpha}\left(t_{0}\right)<v_{\beta}\left(t_{0}\right)$ and hence, $c_{1} \leq c_{2}$. (Note that if $c_{1}>c_{2}$, then Theorem 5 (b) yields $v_{\alpha}(t)>v_{\beta}(t)$ for $t \in(0, T)$, which is not possible.) The result now follows from Theorem 5(b) for $c_{1}<c_{2}$ and from Theorem 5(c) for $c_{1}=c_{2}$.

Corollary 6 Let $\left\{\alpha_{n}\right\} \subset\left[\alpha_{0}, \infty\right)$ be a convergent sequence and let us denote $\lim _{n \rightarrow \infty} \alpha_{n}=\alpha$. Then $\lim _{n \rightarrow \infty} w_{\alpha_{n}}=w_{\alpha}$ in $C[0, T]$.

Proof The proof is indirect. Assume that the statement of the corollary does not hold. Then there exist $\varepsilon,\left\{t_{n}\right\} \subset[0, T]$ and a subsequence of $\left\{\alpha_{n}\right\}$, we denote it again by $\left\{\alpha_{n}\right\}$, such that

$$
\left|w_{\alpha_{n}}\left(t_{n}\right)-w_{\alpha}\left(t_{n}\right)\right| \geq \varepsilon \quad \text { for } n \in \mathbb{N} \text {. }
$$

Let $\mathbb{N}_{1}=\left\{n \in \mathbb{N}: \alpha_{n} \geq \alpha\right\}$ and $\mathbb{N}_{2}=\left\{n \in \mathbb{N}: \alpha_{n} \leq \alpha\right\}$. Let $\mathbb{N}_{1}$ be countable. Then there exists a decreasing subsequence $\left\{\alpha_{\ell_{n}}\right\}_{n \in \mathbb{N}}$ of $\left\{\alpha_{n}\right\}_{n \in \mathbb{N}_{1}}$. By Theorem 8 , the sequence $\left\{w_{\alpha_{\ell_{n}}}\right\}$ is not increasing on $[0, T]$ and $w_{\alpha \ell_{n}} \geq w_{\alpha}$ on this interval. Using (33) and (42), we obtain $w_{\alpha_{\ell_{n}}}(t)=$ $u_{\alpha_{\ell_{n}}}(t) / t, w_{\alpha}(t)=u_{\alpha}(t) / t$. Since $\lim _{n \rightarrow \infty} u_{\alpha_{\ell_{n}}}=u_{\alpha}$ in $C^{1}[0, T]$ it follows from Theorem 3, note that $w_{\alpha_{\ell_{n}}}(0)=\alpha_{\ell_{n}}$, that $w_{\alpha}(0)=\alpha$ and that $\lim _{n \rightarrow \infty} w_{\alpha_{\ell_{n}}}(t)=w_{\alpha}(t)$ for $t \in[0, T]$. This fact together with the monotonicity of $\left\{w_{\alpha_{\ell_{n}}}\right\}$ and $w_{\alpha} \in C[0, T]$ gives $\lim _{n \rightarrow \infty} w_{\alpha_{\ell_{n}}}=w_{\alpha}$ in $C[0, T]$, which contradicts (43). Hence $\lim _{n \rightarrow \infty} w_{\alpha_{n}}=w_{\alpha}$ in $C[0, T]$.

For $\alpha^{*}>\alpha_{0}$, we now define a set $\mathcal{W}^{*}$,

$$
\mathcal{W}^{*}=\left\{w_{\alpha} \text { from (42) with } v_{\alpha} \in \mathcal{R} \text { satisfying (39): } \alpha_{0} \leq \alpha \leq \alpha^{*}\right\}
$$

Theorem 9 Let $\left(\mathrm{H}_{1}^{0}\right)-\left(\mathrm{H}_{3}^{0}\right)$ hold and let $\alpha_{0}$ be from (38). For each $\alpha^{*}>\alpha_{0}$ the set $\mathcal{W}^{*}$ defined by (44) is compact in $C[0, T]$. 
Proof Consider $\alpha \in\left[\alpha_{0}, \alpha^{*}\right]$. By Theorem 8 we have

$$
w_{\alpha_{0}}(t) \leq w_{\alpha}(t) \leq w_{\alpha^{*}}(t), \quad t \in[0, T]
$$

According to (33), (42), and Theorem 4 we have

$$
\begin{aligned}
& u_{\alpha}(t)=t w_{\alpha}(t), \quad u_{\alpha}^{\prime}(0)=w_{\alpha}(0)=\alpha, \quad u_{\alpha} \in \mathcal{S}, \\
& u_{\alpha_{0}}(t) \leq u_{\alpha}(t) \leq u_{\alpha^{*}}(t), \quad t \in[0, T]
\end{aligned}
$$

Let us denote

$$
c_{\alpha}:=-u_{\alpha}^{\prime}(T) \text { and } \mathcal{S}^{*}:=\bigcup_{\alpha_{0} \leq \alpha \leq \alpha^{*}} \mathcal{S}_{c_{\alpha}} .
$$

Conditions (4) and (46) imply $0 \leq c_{\alpha_{0}} \leq c_{\alpha} \leq c_{\alpha^{*}}$. Consequently, using Theorem 4, we obtain

$$
w_{\alpha} \in \mathcal{W}^{*} \quad \text { if and only if } \quad u_{\alpha} \in \mathcal{S}^{*} \text { for } \alpha \in\left[\alpha_{0}, \alpha^{*}\right]
$$

Consider a sequence $\left\{w_{\alpha_{n}}\right\} \subset \mathcal{W}^{*}$. Then there exists a sequence $\left\{u_{\alpha_{n}}\right\} \subset \mathcal{S}^{*}$ with $u_{\alpha_{n}}=$ $t w_{\alpha_{n}}$. By Lemma $1(\mathrm{f})$, the set $\mathcal{S}^{*}$ is compact in $C^{1}[0, T]$. Therefore, there exists a subsequence $\left\{u_{\alpha_{k}}\right\}$ which converges in $C^{1}[0, T]$ to $\bar{u} \in \mathcal{S}^{*}$. In particular, $\lim _{k \rightarrow \infty} u_{\alpha_{k}}^{\prime}(0)=\bar{u}^{\prime}(0)$. Let us denote $\bar{\alpha}:=\bar{u}^{\prime}(0)$, then we have $\lim _{k \rightarrow \infty} \alpha_{k}=\bar{\alpha} \in\left[\alpha_{0}, \alpha^{*}\right]$. This yields, by Corollary 6 , $\lim _{k \rightarrow \infty} w_{\alpha_{k}}=w_{\bar{\alpha}}$ in $C[0, T]$ and $w_{\bar{\alpha}} \in \mathcal{W}^{*}$. Thus we showed that for any sequence in $\mathcal{W}^{*}$, there exists a uniformly converging subsequence on $[0, T]$ with a limit in $\mathcal{W}^{*}$.

\section{Examples}

Using the open domain Matrab Code bvpsuite, we numerically simulate three model problems in order to illustrate theoretical statements made above.

\subsection{Matlab Code bvpsuite}

The MATLAB ${ }^{\mathrm{TM}}$ software package bvpsui te is designed to solve BVPs in ODEs and differential algebraic equations. The solver routine is based on a class of collocation methods whose orders may vary from 2 to 8 . Collocation has been investigated in context of singular differential equations of first and second order in [24, 25], respectively. This method could be shown to be robust with respect to singularities in time and retains its high convergence order in case that the analytical solution is appropriately smooth. The code also provides an asymptotically correct estimate for the global error of the numerical approximation. To enhance the efficiency of the method, a mesh adaptation strategy is implemented, which attempts to choose grids related to the solution behavior, in such a way that the tolerance is satisfied with the least possible effort. The error estimate procedure and the mesh adaptation work dependably provided that the solution of the problem and its global error are appropriately smooth. ${ }^{\mathrm{b}}$ The code and the manual can be downloaded from http://www.math.tuwien.ac.at/ ewa. For further information see [26]. This software proved useful for the approximation of numerous singular BVPs important for applications; see e.g. [9, 27-29]. 

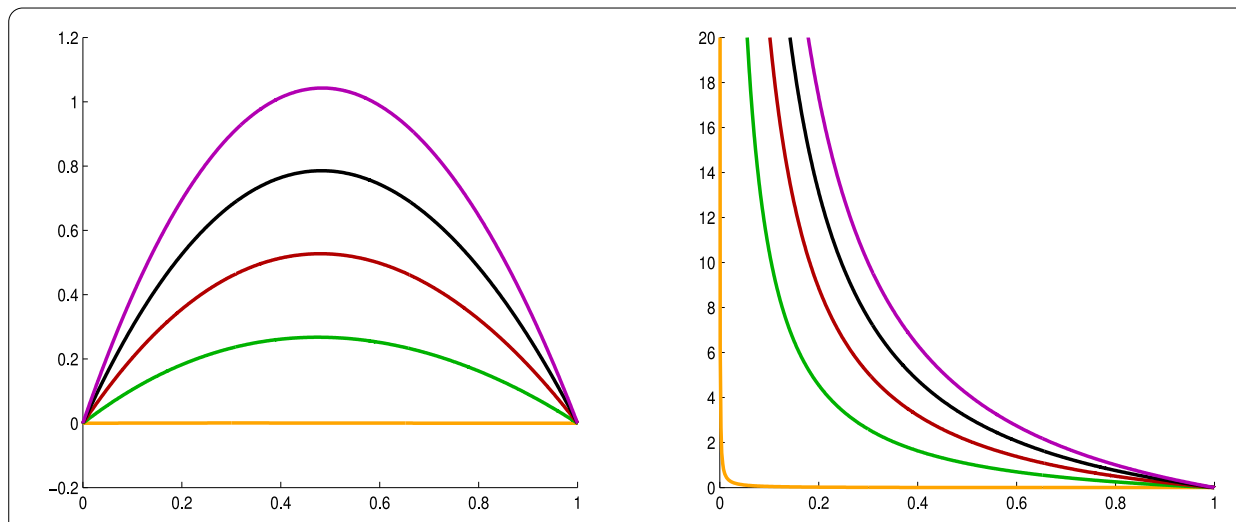

Figure 1 Example 1. Solutions $u(t)$ of (50) (first) and $v(t)$ of (48) (second) for different values of $c, c=0$ orange, $c=1$ green, $c=2$ red, $c=3$ dark blue, $c=4$ magenta. We used a collocation method of order 8 based on Gaussian points and grid adaptation strategy to satisfy the tolerances $T O L_{a b s}=T O L_{r e l}=10^{-7}$.

Example 1 The first example is used to comment on Theorem 5 and to show that if a function $g(t, x)$ in (27) is increasing in $x$, the graphs of positive solutions of problem (27), (28) cannot intersect. Here, we choose

$$
k=2, \quad T=1, \quad \psi(t)=0.01, \quad g(t, x)=x^{2 / 3},
$$

and thus problem (27), (28) becomes

$$
v^{\prime \prime}(t)+\frac{2}{t} v^{\prime}=0.01+v^{\frac{2}{3}}(t), \quad v(0)=\infty, \quad v(1)=0 .
$$

Using substitution (33), which has the form

$$
a=-2, \quad u(t)=t^{2} v(t)
$$

we transform (48) onto the form, $c f$. (34),

$$
u^{\prime \prime}(t)-\frac{2}{t} u^{\prime}(t)+\frac{2}{t^{2}} u(t)=t^{2}\left(0.01+\left(t^{-2} u(t)\right)^{\frac{2}{3}}\right),
$$

and we solve the terminal value problem given by

$$
u^{\prime \prime}(t)-\frac{2}{t} u^{\prime}(t)+\frac{2}{t^{2}} u(t)=0.01 t^{2}+t^{\frac{2}{3}} u^{\frac{2}{3}}(t), \quad u(1)=0, \quad u^{\prime}(1)=-c,
$$

where $c=0,1,2,3$, and 4 . Then, using (49), we recalculate these solution to their blow-up form and obtain different positive solutions $v$ of problem (48). Note that their graphs are ordered after $c, c f$. Figure 1 .

Example 2 We designed this example to see if the condition $\left(\mathrm{H}_{2}^{0}\right)$ is essential for statement (b) of Theorem 5. To this aim, we choose a function $g(t, x)$ which is decreasing in $x$ in (27). Let

$$
k=2, \quad T=1, \quad \psi(t)=0.01, \quad g(t, x)=\frac{1}{1+x} .
$$



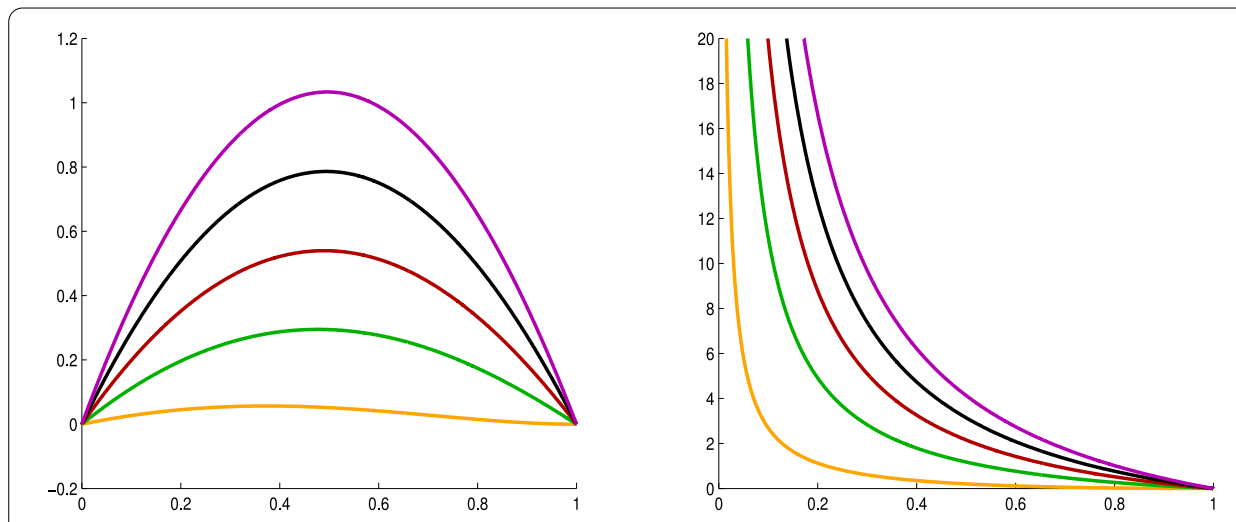

Figure 2 Example 2. Solutions $u(t)$ of (52) (first) and $v(t)$ of (51) (second) for different values of $c, c=0$ orange, $c=1$ green, $c=2$ red, $c=3$ dark blue, $c=4$ magenta. We used a collocation method of order 8 based on Gaussian points and grid adaptation strategy to satisfy the tolerances $T O L_{a b s}=T O L_{r e l}=10^{-8}$.

Then problem (27), (28) has the form

$$
v^{\prime \prime}(t)+\frac{2}{t} v^{\prime}=0.01+\frac{1}{1+v(t)}, \quad v(0)=\infty, \quad v(1)=0 .
$$

Using substitution (49), we rewrite (51) and have

$$
u^{\prime \prime}(t)-\frac{2}{t} u^{\prime}(t)+\frac{2}{t^{2}} u(t)=t^{2}\left(0.01+\frac{1}{1+t^{-2} u(t)}\right) .
$$

The resulting terminal value problem reads

$$
u^{\prime \prime}(t)-\frac{2}{t} u^{\prime}(t)+\frac{2}{t^{2}} u(t)=0.01 t^{2}+\frac{t^{4}}{t^{2}+u(t)}, \quad u(1)=0, \quad u^{\prime}(1)=-c,
$$

where $c=0,1,2,3$, and 4 . Then, using (49), we obtain different positive blow-up solutions of problem (51). However, their graphs are again ordered; see Figure 2. Hence, condition $\left(\mathrm{H}_{2}^{0}\right)$ seems not to be necessary for statement (b) of Theorem 5 to hold. On the other hand, the question, if another model with a decreasing nonlinearity $g$ exists, where solutions cross each other and are not ordered after $c$, remains open.

Example 3 Since, Example 2 did not provide the solution crossings, we constructed another model problem, where in (27) the function $g(t, x)$ oscillates in $x$. Let

$$
k=2, \quad T=1, \quad \psi(t)=0.01, \quad g(t, x)=10\left(1+\sin \left(10 t^{2} x\right)\right) ;
$$

then problem (27), (28) has the form

$$
v^{\prime \prime}(t)+\frac{2}{t} v^{\prime}=10.01+10 \sin \left(10 t^{2} v(t)\right), \quad v(0)=\infty, \quad v(1)=0 .
$$

Using (49), we again transform (53) and solve the terminal value problem

$$
u^{\prime \prime}(t)-\frac{2}{t} u^{\prime}(t)+\frac{2}{t^{2}} u(t)=t^{2}(10.01+10 \sin (10 u(t))), \quad u(1)=0, \quad u^{\prime}(1)=-c,
$$



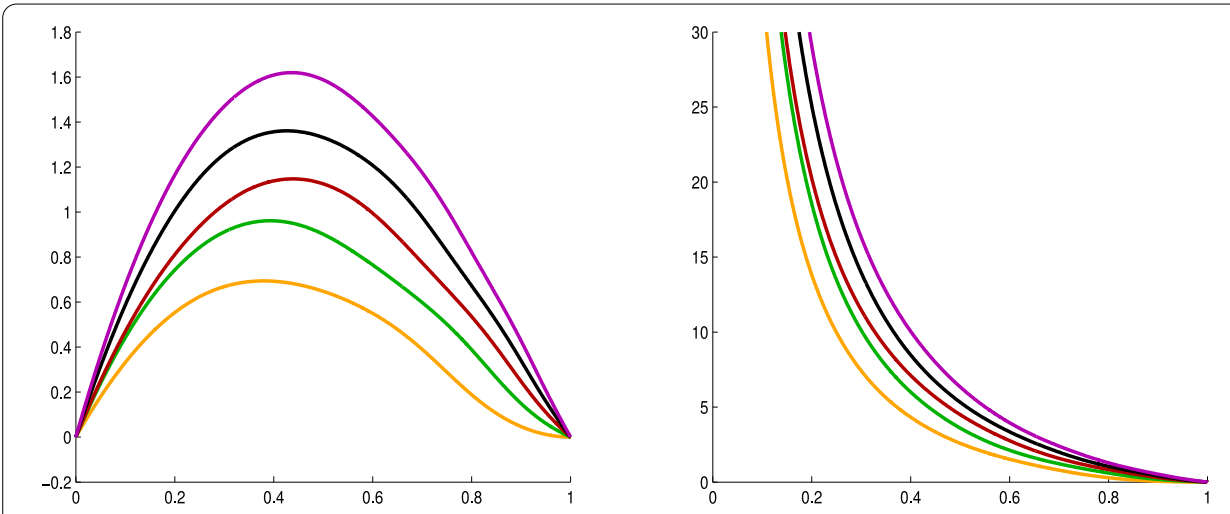

Figure 3 Example 3. Solutions $u(t)$ of (54) (first) and $v(t)$ of (53) (second) for different values of $c, c=0$ orange, $c=1$ green, $c=2$ red, $c=3$ dark blue, $c=4$ magenta. We used a collocation method of order 8 based on Gaussian points and grid adaptation strategy to satisfy the tolerances $T O L_{a b s}=T O L_{r e l}=10^{-9}$.

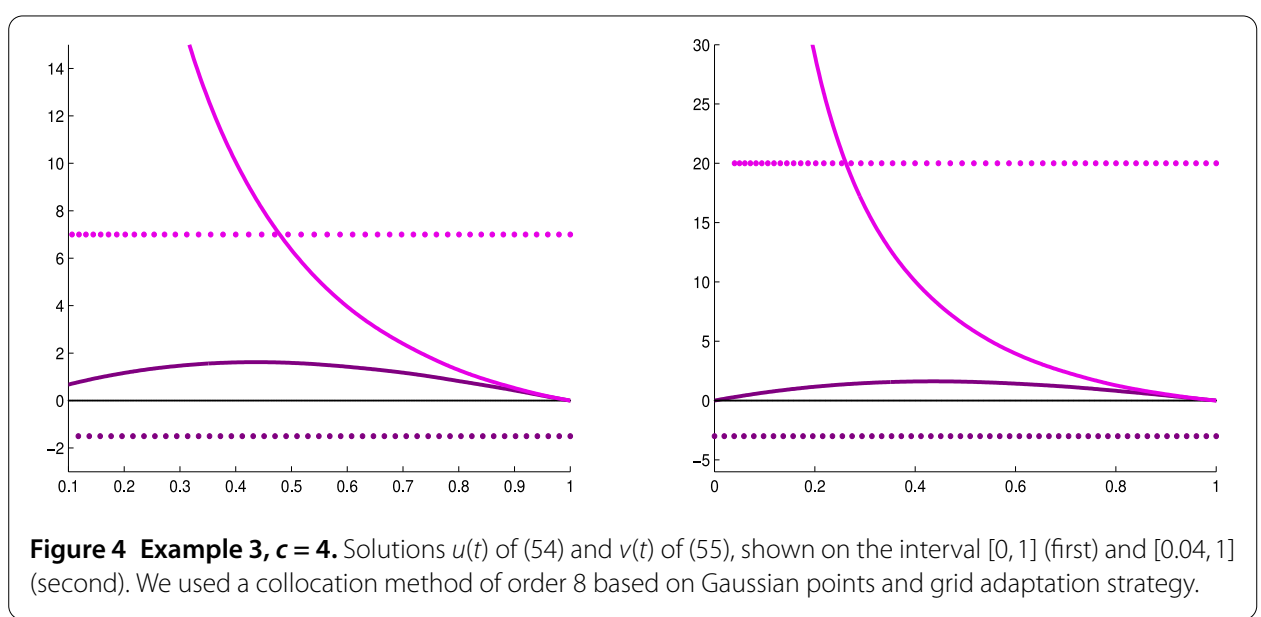

where $c=0,1,2,3$, and 4 . Then, using (49), we calculate the related positive blow-up solutions of problem (53). As can be seen in Figure 3, their graphs are again ordered after $c$ and the question of solution crossings remains open even if we consider oscillating nonlinearities.

To show the advantage of the grid adaptation, we carried out the following additional test. For $c=4$, we evaluated the solution $u$ at the point $t=0.04$ and solved the boundary value problem

$$
v^{\prime \prime}(t)+\frac{2}{t} v^{\prime}=10.01+10 \sin \left(10 t^{2} v(t)\right), \quad v(0.04):=\frac{u(0.04)}{0.04^{2}}, \quad v(1)=0 .
$$

In Figure 4, solutions $u(t)$ of (54) and $v(t)$ of (55), together with the final grids are shown on the interval $[0,1]$ (left) and [0.04,1] (right). Due to the different smoothness of $u$ and $v$, their accuracy strongly varies. While the absolute and relative errors of $u$ (or rather their estimates) on the grid with 52 grid points is $10^{-13}$, the absolute and relative errors of $v$ on the grid with exactly the same number of points is only $10^{-9}$. Note that the code has automatically adapted the location of the grid points to correctly reflect the solution behavior. 


\section{Conclusions}

We have described the set $\mathcal{R}$ of all positive solutions of problem (1), (2), where $h$ has the form $h(t, x)=\psi(t)+g(t, x)$ and assumptions $\left(\mathrm{H}_{1}^{0}\right)-\left(\mathrm{H}_{3}^{0}\right)$ hold. By Theorem 5 we know that $\mathcal{R}$ is nonempty and that for each $c \geq 0$ there exists at least one function $v \in \mathcal{R}$ fulfilling $v^{\prime}(T)=-c$. In addition, graphs of functions from $\mathcal{R}$ do not intersect and $\mathcal{R}$ has a minimal element $v_{0, \min }$.

If we choose an arbitrary $c^{*}>0$ and denote $\mathcal{R}^{*}=\left\{v \in \mathcal{R}:-c^{*} \leq v^{\prime}(T) \leq 0\right\}$, then there exists a maximal element $v_{c^{*} \text {,max }}$ in $\mathcal{R}^{*}$. Clearly $v_{0, \text { min }}^{\prime}(T)=0$ and $v_{c^{*}, \max }^{\prime}(T)=-c^{*}$. According to Theorem 6 , the interior of the set $\left\{(t, x) \in \mathbb{R}^{2}: 0 \leq t \leq T, v_{0, \min }(t) \leq x \leq v_{c^{*}, \max }(t)\right\}$ is fully covered by graphs of functions from $\mathcal{R}$. Finally, we deduce from Theorems 7-9 that the set $\left\{t^{k-1} v: v \in \mathcal{R}^{*}\right\}$ is compact in $C[0, T]$.

Example 1 illustrates the results of Section 3 and hence, according to $\left(\mathrm{H}_{2}^{0}\right)$, we have chosen the increasing function $g(x)=x^{2 / 3}$ in (48). Figure 1 shows ordered graphs of solutions.

In contrast to this, in Example 2 and Example 3, we have chosen the decreasing function $g(x)=1 /(1+x)$ in (51) and the non-monotonous function $g(t, x)=10\left(1+\sin \left(10 t^{2} x\right)\right)$ in (53), respectively. We can see on Figure 2 and Figure 3 that the graphs of solutions are ordered in both cases. But to prove such order of solutions for non-increasing $g$ is an open problem. On the other hand, the question of the construction of problem (27), (28) whose positive solutions cross each other remains open, as well.

Competing interests

The authors declare that they have no competing interests.

Authors' contributions

The authors read and approved the final draft. IR and SS contributed to the analytical part of the paper and JG and EW contributed to the numerical part of the paper.

\section{Author details}

'Department for Analysis and Scientific Computing, Vienna University of Technology, Wiedner Hauptstraße 8-10, Wien, A-1040, Austria. 'Department of Mathematics, Faculty of Science, Palacký University, 17. listopadu 12, Olomouc, 77146, Czech Republic.

\section{Acknowledgements}

This research was supported by the grants IGA PrF_2013_013 and IGA-PrF_2014028. The authors are grateful to the referees for useful comments and suggestions which improved the paper.

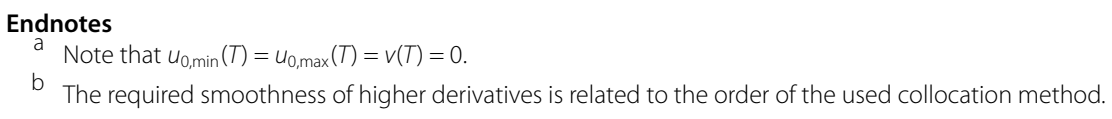

Received: 13 December 2013 Accepted: 5 May 2014 Published: 16 May 2014

\section{References}

1. Bongiorno, V, Scriven, LE, Davis, HT: Molecular theory of fluid interfaces. J. Colloid Interface Sci. 57, $462-475$ (1967)

2. Gouin, H, Rotoli, H: An analytical approximation of density profile and surface tension of microscopic bubbles for van der Waals fluids. Mech. Res. Commun. 24, 255-260 (1997)

3. van der Waals, JD, Kohnstamm, R: Lehrbuch der Thermodynamik, Vol. 1. Maas \& von Suchtelen, Leipzig (1908)

4. Fife, PC: Mathematical Aspects of Reacting and Diffusing Systems. Lecture Notes in Biomathematics, vol. 28. Springer, New York (1979)

5. Fischer, RA: The wave of advance of advantageous genes. Ann. Eugenics 7, 355-369 (1937)

6. Abraham, FF: Homogeneous Nucleation Theory. Academic Press, New York (1974)

7. Linde, AP: Particle Physics and Inflationary Cosmology. Harwood Academic, Chur (1990)

8. Derrick, GH: Comments on nonlinear wave equations as models for elementary particles. J. Math. Phys. 5, 1252-1254 (1965)

9. Kitzhofer, G, Koch, O, Lima, P, Weinmüller, EB: Efficient numerical solution of the density profile equation in hydrodynamics. J. Sci. Comput. 32, 411-424 (2007)

10. Lima, PM, Chemetov, NV, Konyukhova, NB, Sukov, Al: Analytical-numerical investigation of bubble-type solutions of nonlinear singular problems. J. Comput. Appl. Math. 189, 260-273 (2006)

11. Rachůnková, I, Koch, O, Pulverer, G, Weinmüller, EB: On a singular boundary value problem arising in the theory of shallow membrane caps. J. Math. Anal. Appl. 332, 523-541 (2007) 
12. Rachůnková, I, Staněk, S, Weinmüller, EB, Zenz, M: Neumann problems with time singularities. Comput. Math. Appl. $60,722-733(2010)$

13. Rachůnková, I, Tomeček, J: Bubble-type solutions of nonlinear singular problems. Math. Comput. Model. 51, 658-669 (2010)

14. Astashova, l: On power and non-power asymptotic behavior of positive solutions to Emden-Fowler type higher-order equations. Adv. Differ. Equ. 2013(220), 1-15 (2013)

15. Karls, M, Mohammed, A: Integrability of blow-up solutions to some non-linear differential equations. Electron. J. Differ. Equ. 33, 1-8 (2004)

16. Kiguradze, IT, Chanturia, TA: Asymptotic Properties of Solutions of Nonautonomous Ordinary Differential Equations. Kluwer Academic, London (1993)

17. Jaroš, J, Kusano, T: On black hole solutions of second order differential equations with a singular nonlinearity in the differential operator. Funkc. Ekvacioj 43, 491-509 (2000)

18. Jaroš, J, Kusano, T: On white hole solutions of a class of nonlinear ordinary differential equations of the second order. Funkc. Ekvacioj 45, 319-339 (2002)

19. Tanigawa, $\mathrm{T}$ : On the structure of positive solutions of a class of fourth order nonlinear differential equations. Ann. Mat. Pura Appl. 185, 521-536 (2006)

20. Rachůnková, I, Staněk, S: Properties of the set of positive solutions to Dirichlet boundary value problems with time singularities. Cent. Eur. J. Math. 11, 112-132 (2013)

21. Kiguradze, IT, Shekhter, BL: Singular boundary value problems for second-order ordinary differential equations. J. Sov. Math. 43, 2340-2417 (1988)

22. Lomtatidze, A, Malaguti, L: On a two-point boundary value problem for the second order differential equations with singularities. Nonlinear Anal. 52, 1553-1567 (2003)

23. Rachůnková, I, Spielauer, A, Staněk, S, Weinmüller, EB: Positive solutions of nonlinear Dirichlet BVPs in ODEs with time and space singularities. Bound. Value Probl. 2013(6), 1-22 (2013)

24. de Hoog, F, Weiss, R: Collocation methods for singular boundary value problems. SIAM J. Numer. Anal. 15, 198-217 (1978)

25. Weinmüller, EB: Collocation for singular boundary value problems of second order. SIAM J. Numer. Anal. 23, 1062-1095 (1986)

26. Kitzhofer, G, Pulverer, G, Simon, C, Koch, O, Weinmüller, EB: The new matlab solver bvpsuite for the solution of singular implicit BVPS. J. Numer. Anal. Ind. Appl. Math. 5, 113-134 (2010)

27. Budd, C, Koch, O, Weinmüller, EB: From nonlinear PDEs to singular ODEs. Appl. Numer. Math. 56, 413-422 (2006)

28. Hammerling, R, Koch, O, Simon, C, Weinmüller, EB: Numerical solution of eigenvalue problems in electronic structure computations. J. Comp. Physiol. 181, 1557-1561 (2010)

29. Rachůnková, I, Pulverer, G, Weinmüller, EB: A unified approach to singular problems arising in the membrane theory. Appl. Math. 55, 47-75 (2010)

10.1186/1687-2770-2014-121

Cite this article as: Gschwindl et al.: Positive blow-up solutions of nonlinear models from real world dynamics.

Boundary Value Problems 2014, 2014:121

\section{Submit your manuscript to a SpringerOpen ${ }^{\circ}$ journal and benefit from:}

- Convenient online submission

Rigorous peer review

- Immediate publication on acceptance

- Open access: articles freely available online

- High visibility within the field

- Retaining the copyright to your article 